\title{
Large-Time Behavior of the Broadwell Model of a Discrete Velocity Gas
}

\author{
J. Thomas Beale ${ }^{\star}$ \\ Department of Mathematics, Duke University, Durham, NC 27706, USA
}

\begin{abstract}
We study the behavior of solutions of the one-dimensional Broadwell model of a discrete velocity gas. The particles have velocity \pm 1 or 0 ; the total mass is assumed finite. We show that at large time the interaction is negligible and the solution tends to a free state in which all the mass travels outward at speed 1 . The limiting behavior is stable with respect to the initial state. No smallness assumptions are made.
\end{abstract}

\section{Introduction}

Broadwell [1] studied the structure of shocks in a model of rarefied gas in which the particles travel with speed $c$ in either direction along a coordinate axis. If particles traveling in opposite directions collide, they are equally likely to move in each of the three coordinate directions after collision, with velocities of opposite sign. Other collisions can only lead to an exchange of velocities. If $N_{1}^{+}(x, y, z, t)$ is the density of particles with velocity $(c, 0,0), N_{1}^{-}$the density with velocity $(-c, 0,0)$, and similarly for $N_{2}^{ \pm}, N_{3}^{ \pm}$, the resulting equations are

$$
\begin{aligned}
& D_{t} N_{1}^{+}+c D_{x} N_{1}^{+}=\frac{\sigma}{3}\left(N_{2}^{+} N_{2}^{-}+N_{3}^{+} N_{3}^{-}-2 N_{1}^{+} N_{1}^{-}\right), \\
& D_{t} N_{1}^{-}-c D_{x} N_{1}^{-}=\frac{\sigma}{3}\left(N_{2}^{+} N_{2}^{-}+N_{3}^{+} N_{3}^{-}-2 N_{1}^{+} N_{1}^{-}\right),
\end{aligned}
$$

etc., where $\sigma$ is the frequency of collision. (Such discrete velocity models of a gas were introduced by Maxwell; see [5] for a survey.)

Here we consider the special case of one-dimensional motions in which the $N$ 's are independent of $y, z$, and furthermore $N_{2}^{+}=N_{2}^{-}=N_{3}^{+}=N_{3}^{-}$. Setting $N_{1}^{+}=v(x, t)$, $N_{1}^{-}=w(x, t), N_{2}^{+}=z(x, t)$ and rescaling the variables so that $c=1, \sigma=3 / 2$, we can

^ Partially supported by N.S.F. Grant No. NSF-DMS-84-08393 
write the equations as

$$
\begin{aligned}
v_{t}+v_{x} & =z^{2}-v w, \\
w_{t}-w_{x} & =z^{2}-v w, \\
z_{t} & =\frac{1}{2}\left(v w-z^{2}\right) .
\end{aligned}
$$

Suppose in addition that initial data are prescribed:

$$
v(x, 0)=v_{0}(x), \quad w(x, 0)=w_{0}(x), \quad z(x, 0)=z_{0}(x) .
$$

If the data are nonnegative, the same is true for the solution. Moreover, for integrable data, the mass and momentum

$$
\int_{-\infty}^{\infty}(v+w+4 z) d x, \quad \int_{-\infty}^{\infty}(v-w) d x
$$

are conserved in time. An equivalent statement is that the partial masses

$$
m^{+}=\int_{-\infty}^{\infty}(v+2 z) d x, \quad m^{-}=\int_{-\infty}^{\infty}(w+2 z) d x,
$$

are conserved. Since the interaction terms are quadratic in the unknowns, it is not immediately apparent that solutions continue indefinitely. However, it was shown by Crandall and Tartar [14] that regular solutions of the initial value problem (1.1), (1.2) exist for all time for suitable initial data, in particular if the data are uniformly bounded and integrable. This argument relied on an earlier existence result of Nishida and Mimura [13] for sufficiently small mass. (The properties of solutions and the existence theory are reviewed in Sect. 2.) The purpose of this paper is to describe the behavior at large time for solutions of finite mass. We show that the solution evolves toward a state in which $v$ and $w$ have become waves traveling outward with velocity \pm 1 without interaction, and $z$ is zero. Thus, while the interactions may produce local growth, the ultimate nature of the solution is determined by the spatial spreading at different velocities.

The system (1.1) is one of a variety of possible one-dimensional models of a discrete velocity gas. Mathematical questions for a general class of such systems were treated in [15]. For the Carleman equations $v_{t}+v_{x}=w^{2}-v^{2}, w_{t}-w_{x}=v^{2}-$ $w^{2}$, the maximum norm of the solution is nonincreasing. Using this fact Illner and Reed [6] showed that bounded solutions decay uniformly at large time (see also [15, Appendix 2]). For other systems such as the Broadwell Eq. (1.1), asymptotic descriptions have been given only for small data [15]. For (1.1) the maximum norm may increase, and there do not seem to be any natural norms other than the mass which are conserved or nonincreasing. Our strategy here is first to obtain a weak decay statement for $v$ and $w$, and then to show using this that a quantity related to the $L^{p}$ norm is eventually nonincreasing. The nonnegativity of solutions is important for our method. Other work on the existence and basic properties of (1.1) and related systems includes [2,3,7-15].

We now summarize the results. We shall say that the initial data $v_{0}, w_{0}, z_{0}$ are admissible if they are nonnegative, continuously differentiable, uniformly bounded, and integrable. In the statements below, the data are assumed to satisfy these conditions. The number $p$ is arbitrary with $1 \leqq p<\infty$. 
Theorem 1. (Boundedness) For any solution there exists a constant $K$ so that

$$
|v(x, t)|,|w(x, t)|,|z(x, t)| \leqq K
$$

for $x \in \mathbb{R}, t \geqq 0$.

Theorem 2. ( $\boldsymbol{L}^{p}$ asymptotics) $A s t \rightarrow \infty, z(\cdot, t) \rightarrow 0$ in $L^{p}(\mathbb{R}) ; v(\cdot+t, t)$ converges in $L^{p}$ to a limit $v^{\infty}$; and $w(\cdot-t, t)$ converges in $L^{p}$ to a limit $w^{\infty}$. In particular, the total mass inside any finite interval goes to zero as $t \rightarrow \infty$. The limiting states are uniformly bounded by $K$ and satisfy

$$
\int_{-\infty}^{\infty} v^{\infty} d x=m^{+}, \quad \int_{-\infty}^{\infty} w^{\infty} d x=m^{-}
$$

Theorem 3. ( $\boldsymbol{L}^{p}$ Stability) Let $\bar{v}, \bar{w}, \bar{z}$ be a solution with initial data $\bar{v}_{0}, \bar{w}_{0}, \bar{z}_{0}$. Given $\varepsilon>0$, if $\left(v_{0}, w_{0}, z_{0}\right)$ are sufficiently close to $\left(\bar{v}_{0}, \bar{w}_{0}, \bar{z}_{0}\right)$ in $L^{1}(\mathbb{R})$ and $L^{\infty}(\mathbb{R})$, then the solution satisfies

$$
|v(\cdot, t)-\bar{v}(\cdot, t)|_{L^{p}},|w(\cdot, t)-\bar{w}(\cdot, t)|_{L^{p}},|z(\cdot, t)-\bar{z}(\cdot, t)|_{L^{p}}<\varepsilon
$$

uniformly in $t$, and consequently

$$
\left|v^{\infty}-\bar{v}^{\infty}\right|_{L^{p}}, \quad\left|w^{\infty}-\bar{w}^{\infty}\right|_{L^{p}}<\varepsilon .
$$

Theorem 4. (Boundedness in a Neighborhood) For any solution $\bar{v}, \bar{w}, \bar{z}$, there is a constant $K^{\prime}$ so that, for $\left(v_{0}, w_{0}, z_{0}\right)$ close enough to $\left(\bar{v}_{0}, \bar{w}_{0}, \bar{z}_{0}\right)$ in $L^{1}(\mathbb{R}) \cap L^{\infty}(\mathbb{R})$,

$$
|v(x, t)|,|w(x, t)|,|z(x, t)| \leqq K^{\prime}
$$

for $x \in \mathbb{R}, t \geqq 0$.

Theorem 5. (Uniform Asymptotics) Assume that $v_{0}, w_{0}, z_{0}$ tend to zero as $x \rightarrow \pm \infty$. Then $z(x, t) \rightarrow 0$ uniformly in $x$ as $t \rightarrow \infty$, and $v(x, t), w(x, t) \rightarrow 0$ uniformly for $x$ in any finite interval. The limiting functions $v^{\infty}, w^{\infty}$ are continuous and tend to zero as $x \rightarrow \pm \infty$. Moreover, the convergence of $v(\cdot+t, t), w(\cdot-t, t)$ to $v^{\infty}, w^{\infty}$ as $t \rightarrow \infty$ is uniform in $x$.

In Sect. 2 we recall the basic properties of solutions, outline the proof of the existence theorem, and derive the weak local decay statement for $v, w$ (Lemmas 2.1, 2.2). We prove Theorem 1 in Sect. 3. Introducing weight functions related to the mass integrals, we show that a weighted $L^{p}$ norm is non-increasing at large time. Letting $p \rightarrow \infty$, we have a uniform bound. The same estimate gives a weak decay statement for $z$. Theorems 2-4 are proved in Sect. 4. We first show (Lemma 4.2) that the interaction terms are integrable in space-time. As noted in [15], the asymptotic description in $L^{1}$ is a simple consequence of this statement. Theorems 3 and 4 are obtained by sharpening the proofs of Lemma 4.2 and Theorem 1. In Sect. 5 we first show that $z$ decays uniformly by comparing with an ordinary differential equation. We then complete the proof of Theorem 5 following an argument of Tartar [15].

There is a surprising variety in the behavior of the different particle systems. As observed by Tartar [15], the Carleman model cannot have $L^{1}$ limits as in Theorem 2; since the solutions decay in $L^{\infty}$, the limit would have to be zero, but this is impossible because of conservation of mass. A system similar to (1.1) is obtained by setting $N_{1}^{+}=v, N_{1}^{-}=w, N_{2}^{+}=N_{3}^{+}=z^{+}, N_{2}^{-}=N_{3}^{-}=z^{-}$. The resulting system, which is 
slightly more general than (1.1) and was treated in [14], is

$$
\begin{aligned}
v_{t}+v_{x} & =z^{+} z^{-}-v w, \quad w_{t}-w_{x}=z^{+} z^{-}-v w, \\
z_{t}^{+} & =\frac{1}{2}\left(v w-z^{+} z^{-}\right), \quad z_{t}^{-}=\frac{1}{2}\left(v w-z^{+} z^{-}\right) .
\end{aligned}
$$

It is not difficult to modify the proof of Theorem 1 to show that solutions of this system remain bounded. However, the direct analogue of Theorem 2 cannot be true: since $z_{t}^{+}=z_{t}^{-}, z^{+}-z^{-}$is constant in time, and both $z$ 's cannot approach zero if this difference is initially nonzero. It will be evident that the techniques introduced here can be applied to related systems of equations. Applications to a general class of semilinear systems will be developed in forthcoming work.

\section{Background and Weak Decay}

We first discuss a few basic properties of solutions. Suppose we have a $C^{1}$ solution on the closure of a trapezoidal region $S=\{(x, t): 0<t<T, a+t<x<b-t\}$. If $a=$ $-\infty$ or $b=\infty$, we assume that the solution is uniformly bounded. Three elementary properties are

(i) (Uniqueness) There is only one solution with prescribed initial data.

(ii) (Domain of Dependence) For $\left(x_{0}, t_{0}\right) \in S$, the solution at $\left(x_{0}, t_{0}\right)$ depends only on the initial data on the interval $x_{0}-t_{0} \leqq x \leqq x_{0}+t_{0}$.

(iii) (Nonnegativity) If $v_{0}, w_{0}, z_{0} \geqq 0$, then $v, w, z \geqq 0$ throughout $S$.

The solution can be constructed locally in time by a standard argument (e.g., [4, Chap. V, §6]). The equations are written in integral form along characteristics and the solution is obtained as the fixed point of a contraction mapping. The domain of dependence property is evident from this construction. Because the fixed point is unique, two solutions with the same initial data must agree on a short time interval, and this local uniqueness statement implies property (i). Finally, to verify (iii), it is not difficult to modify the contraction mapping argument mentioned above so that nonnegativity is preserved under the iteration, and thus the solution obtained in the limit must be nonnegative (e.g., see Theorem 2 of [14] or (4.12) of [15]).

If $S=\mathbb{R} \times(0, T)$ and the initial data is admissible in the sense of Sect. 1 , and in particular in $L^{1}(\mathbb{R})$, the conservation law

$$
(v+w+4 z)_{t}+(v-w)_{x}=0,
$$

implies that the solution is in $L^{1}(\mathbb{R})$ for each $t$ and

$$
\int_{-\infty}^{\infty}(v+w+4 z) d x=m
$$

representing the total mass, is constant in time. Similarly

$$
(v-w)_{t}+(v+w)_{x}=0,
$$

gives the conservation of momentum. An equivalent pair of conservation laws is

$$
\begin{gathered}
(v+2 z)_{t}+v_{x}=0, \\
(w+2 z)_{t}-w_{x}=0,
\end{gathered}
$$


and these lead to (1.3).

The fundamental existence theorem is the following.

Existence Theorem. Given admissible initial data $v_{0}, w_{0}, z_{0}$ on $\mathbb{R}$, there exists a unique $C^{1}$ solution of Eq. (1.1) defined on $\mathbb{R} \times(0, \infty)$ which is uniformly bounded on $\mathbb{R} \times[0, T]$ for each $T$.

Since the solution is in $L^{1} \cap L^{\infty}(\mathbb{R})$ for each $t$, it is also in $L^{p}(\mathbb{R})$ for every $p \geqq 1$. This theorem was proved by Crandall and Tartar $[14,15]$ using an existence theorem for small mass due to Nishida and Mimura [13]. We outline the proof for the sake of completeness. An argument of Nishida and Mimura shows by an integration along characteristics that if $m<2$ and the initial data are bounded by $K_{0}$, the solution must be bounded by $K=2 K_{0} /(2-m)$. Thus if $m \leqq 1$, we have $v, w, z \leqq 2 K_{0}$. A uniform bound on the solution easily leads to an exponential bound on the derivatives. The contraction mapping argument produces a $C^{1}$ solution for a short time, and if $m \leqq 1$, the uniform bounds allow us to construct a solution for all time in successive steps.

Now, following Crandall and Tartar, we consider the initial value problem with data of arbitrary mass. Let $K_{0}$ be the maximum for the initial data. We construct a solution in $S=\left\{(x, t): 0<t<T,\left|x-x_{0}\right|<2 T-t\right\}$, where $x_{0}$ is an arbitrary point. Since the solution in this region is independent of the data for $\left|x-x_{0}\right|>2 T$, we may replace $v_{0}, w_{0}, z_{0}$ by data vanishing outside $\left|x-x_{0}\right|<3 T$ without affecting the solution; this can be done without increasing $m$ or $K_{0}$. We now consider the initial value problem for $x \in \mathbb{R}$ with this modified data. Since by (ii) the solution has compact support for each $t$, the entropy function

$$
\int_{-\infty}^{\infty}(v \log v+w \log w+4 z \log z) d x
$$

is defined, and it is easily seen to be nonincreasing in $t$. Since $u \log u \geqq-1 / e$ for $u \geqq 0$, it follows that

$$
\int_{-\infty}^{\infty}\left(v \log ^{+} v+w \log ^{+} w+4 z \log ^{+} z\right) d x \leqq 36 T K_{0} \log K_{0}+48 T / e
$$

for $t \leqq T$ or as long as the solution is defined. Here $\log ^{+} u$ means the positive part of $\log u$. As a consequence, it can be shown that there exists a number $r>0$, depending only on $K_{0}$ and $T$, so that, as long as the solution is defined,

$$
\int_{I}[v(x, t)+w(x, t)+4 z(x, t)] d x<1,
$$

for every interval $I$ of length $\leqq r$ with $I \times\{t\} \subseteq S$. (See [15], proof of Theorem 4.) As a result we can build the solution in $S$ by applying the small mass existence result on intervals of length $r$ in time steps of fixed size, say $r / 3$. Solutions agree on overlaps by uniqueness. At each time step the maximum increases at most by a factor of length 2 . By varying $x_{0}$ we construct a $C^{1}$ solution on $\mathbb{R} \times[0, T]$, the number $r$ remaining unchanged. Because of conservation of mass, the solution is $L^{1}$ for each $t$. Finally, since $T$ was arbitrary, the theorem is proved.

We now derive two weak decay statements for $v, w$ at large time. They depend 
only on the conservation laws (2.4), (2.5) and the nonnegativity property.

Lemma 2.1. For each $x$,

$$
\int_{0}^{\infty} v(x, t) d t \leqq m, \quad \int_{0}^{\infty} w(x, t) d t \leqq m .
$$

Proof. We apply the divergence theorem to (2.4) on the region $S=\left\{\left(x^{\prime}, t^{\prime}\right):-\infty<\right.$ $\left.x^{\prime}<x, 0<t^{\prime}<t\right\}$, obtaining

$$
\int_{0}^{t} v\left(x, t^{\prime}\right) d t^{\prime}+\int_{-\infty}^{x}(v+2 z)\left(x^{\prime}, t\right) d x^{\prime}=\int_{-\infty}^{x}\left(v_{0}+2 z_{0}\right) d x^{\prime} .
$$

(Because $v \in L^{1}(S)$, there is no problem in letting $x^{\prime} \rightarrow-\infty$.) Since each term is nonnegative and the right side is part of the mass, we have

$$
\int_{0}^{t} v\left(x, t^{\prime}\right) d t^{\prime} \leqq m
$$

and the conclusion follows. The case of $w$ is similar with (2.6) replaced by the identity

$$
\int_{0}^{t} w\left(x, t^{\prime}\right) d t^{\prime}+\int_{x}^{\infty}(w+2 z)\left(x^{\prime}, t\right) d x^{\prime}=\int_{x}^{\infty}\left(w_{0}+2 z_{0}\right) d x^{\prime} .
$$

Lemma 2.2. For every $L$ and every $\varepsilon>0$, there exists $T(\varepsilon)$ so that for $t \geqq T(\varepsilon)$, we have

$$
\int_{t}^{\infty}(v+w)\left(x, t^{\prime}\right) d t^{\prime} \leqq \varepsilon
$$

uniformly for $-L \leqq x \leqq L$.

Proof. We use the momentum conservation equation (2.3). For $|x| \leqq L$, the divergence theorem applied on the rectangle $\left\{x<x^{\prime}<L, t<t^{\prime}<T\right\}$ gives

$$
\begin{aligned}
& \int_{t}^{T}(v+w)\left(x, t^{\prime}\right) d t^{\prime}-\int_{t}^{T}(v+w)\left(L, t^{\prime}\right) d t^{\prime} \\
& =\int_{x}^{L}(v-w)\left(x^{\prime}, T\right) d x^{\prime}-\int_{x}^{L}(v-w)\left(x^{\prime}, t\right) d x^{\prime} .
\end{aligned}
$$

Now since

$$
\int_{0}^{\infty} \int_{-L}^{L}(v+w) d x^{\prime} d t^{\prime} \leqq 4 L m
$$

by Lemma 2.1 , there is a sequence $T_{n} \rightarrow \infty$ so that

$$
\int_{-L}^{L}(v+w)\left(x^{\prime}, T_{n}\right) d x^{\prime} \rightarrow 0 \quad \text { as } \quad n \rightarrow \infty .
$$

Let $T=T_{n}$ in (2.8) and $n \rightarrow \infty$; the third integral goes to zero, and we obtain

$$
\int_{t}^{\infty}(v+w)\left(x, t^{\prime}\right) d t^{\prime}=\int_{t}^{\infty}(v+w)\left(L, t^{\prime}\right) d t^{\prime}-\int_{x}^{L}(v-w)\left(x^{\prime}, t\right) d x^{\prime}
$$

Suppose now that we replace $t$ by $T_{n}$ from the same sequence as above. For large $n$, 
the last integral is $\leqq \varepsilon / 2$ in magnitude, uniformly in $x$ for $|x| \leqq L$. The middle integral will also be $\leqq \varepsilon / 2$ for $n$ large enough. Thus for some $n$

$$
\int_{t}^{\infty}(v+w)\left(x, t^{\prime}\right) d t^{\prime} \leqq \varepsilon
$$

for $|x| \leqq L$ with $t=T_{n}$, and therefore the same is true for $t>T_{n}$, since the integral is nonincreasing in $t$.

We will also need the following facts, which are evident from the proof of Lemma 2.1.

Lemma 2.3. (a) For each $x$

and

$$
\int_{0}^{\infty} v(x, t) d t \leqq \int_{-\infty}^{x}\left(v_{0}+2 z_{0}\right) d x^{\prime}
$$

$$
\int_{0}^{\infty} w(x, t) d t \leqq \int_{x}^{\infty}\left(w_{0}+2 z_{0}\right) d x^{\prime}
$$

(b) The integrals

$$
\int_{-\infty}^{x}(v+2 z)\left(x^{\prime}, t\right) d x^{\prime}, \quad \int_{x}^{\infty}(w+2 z)\left(x^{\prime}, t\right) d x^{\prime}
$$

are nonincreasing in $t$.

\section{Boundedness of Solutions}

To show that solutions remain uniformly bounded we estimate $L^{p}$ norms and eventually let $p \rightarrow \infty$. With this aim, we first multiply the $v$-equation by $p v^{p-1}$, obtaining

$$
D_{t}\left(v^{p}\right)+D_{x}\left(v^{p}\right)=p v^{p-1} z^{2}-p v^{p} w
$$

We will need to multiply further by a weight function $\mu^{+}$depending on the unknowns. The equation then becomes

$$
D^{+}\left(\mu^{+} v^{p}\right)=\left(D^{+} \mu^{+}\right) v^{p}+p \mu^{+} v^{p-1} z^{2}-p \mu^{+} v^{p} w
$$

where $D^{+}=D_{t}+D_{x}$, with $\mu^{+}$to be chosen. In a similar way we have for $w$ and $z$, with $D^{-}=D_{t}-D_{x}$,

$$
\begin{aligned}
& D^{-}\left(\mu^{-} w^{p}\right)=\left(D^{-} \mu^{-}\right) w^{p}+p \mu^{-} w^{p-1} z^{2}-p \mu^{-} v w^{p} \\
& D_{t}\left(4 \mu^{0} z^{p}\right)=4\left(D_{t} \mu^{0}\right) z^{p}+2 p \mu^{0} z^{p-1} v w-2 p \mu^{0} z^{p+1}
\end{aligned}
$$

Thus

$$
D_{t}\left(\mu^{+} v^{p}+\mu^{-} w^{p}+4 \mu^{0} z^{p}\right)+D_{x}\left(\mu^{+} v^{p}-\mu^{-} w^{p}\right)=R(x, t)
$$

with $R$ the sum of terms on the right in (3.1)-(3.3). We will see that choices can be made so that $R \leqq 0$ at large time, and thus

$$
\int_{-\infty}^{\infty}\left(\mu^{+} v^{p}+\mu^{-} w^{p}+4 \mu^{0} z^{p}\right) d x
$$


is nonincreasing.

We want to choose $\mu^{+}$, for example, so that $D^{+} \mu^{+}$includes a term like $-p z \mu^{+}$, in order to control the growth terms. This can be accomplished by taking the $\mu$ 's to be exponentials of mass integrals. Let

$$
\ell^{-}(x, t)=\int_{-\infty}^{x}(v+w+4 z)\left(x^{\prime}, t\right) d x^{\prime} .
$$

Obviously $D_{x} \ell^{-}=v+w+4 z$. On the other hand, integrating the mass conservation Eq. (2.1) over $\left\{-\infty<x^{\prime}<x, 0<t^{\prime}<t\right\}$ gives

$$
\ell^{-}(x, t)+\int_{0}^{t}(v-w)\left(x, t^{\prime}\right) d t^{\prime}=\int_{-\infty}^{x}\left(v_{0}+w_{0}+4 z_{0}\right) d x^{\prime}
$$

so that $D_{t} \ell^{-}=w-v$. Now let

$$
\ell^{+}(x, t)=m-\ell^{-}(x, t)=\int_{x}^{\infty}(v+w+4 z) d x^{\prime} .
$$

Then $D^{+} \ell^{+}=-4 z-2 w, D^{-} \ell^{-}=-4 z-2 v$. We define $\mu^{+}=\exp p \ell^{+}, \mu^{-}=$ $\exp p \ell^{-}$, so that

$$
D^{+} \mu^{+}=-p(4 z+2 w) \mu^{+}, \quad D^{-} \mu^{-}=-p(4 z+2 v) \mu^{-} .
$$

The $z$-multiplier will be chosen later. We note for future use that $0 \leqq \ell^{ \pm} \leqq m$ and therefore $1 \leqq \mu^{ \pm} \leqq e^{m p}=M^{p}$, where $M=e^{m}$.

In the estimates below we use Young's inequality:

$$
A B \leqq \frac{1}{p} A^{p}+\frac{1}{q} B^{q}, \quad \frac{1}{p}+\frac{1}{q}=1 .
$$

For the growth term in the $v$-equation we set $A=z, B=v^{p-1}$ to obtain

$$
v^{p-1} z^{2}=\left(v^{p-1} z\right) z \leqq \frac{1}{p} z^{p+1}+\frac{p-1}{p} v^{p} z
$$

Substituting (3.5), (3.6) in (3.1), we find

$$
D^{+}\left(\mu^{+} v^{p}\right) \leqq-p \mu^{+}(4 z+2 w) v^{p}+(p-1) \mu^{+} v^{p} z+\mu^{+} z^{p+1}-p \mu^{+} v^{p} w,
$$

and thus

$$
D_{t}\left(\mu^{+} v^{p}\right)+D_{x}\left(\mu^{+} v^{p}\right) \leqq \mu^{+} z^{p+1}-3 p \mu^{+} v^{p} w
$$

In the same way

$$
D_{t}\left(\mu^{-} w^{p}\right)-D_{x}\left(\mu^{-} w^{p}\right) \leqq \mu^{-} z^{p+1}-3 p \mu^{-} v w^{p}
$$

In constructing $\mu^{0}$ we take advantage of Lemma 2.2. We will choose the length $L$ so that

$$
\int_{|x|>L}\left(v_{0}+w_{0}+4 z_{0}\right) d x<\varepsilon_{0} .
$$

Here $\varepsilon_{0}$ is a number depending only on $m$, to be determined below (see (3.21)), and $L$ 
will be fixed. Now define

$$
k^{+}(x, t)=\int_{t}^{\infty} v\left(x, t^{\prime}\right) d t^{\prime}, \quad k^{-}(x, t)=\int_{t}^{\infty} w\left(x, t^{\prime}\right) d t^{\prime},
$$

and

$$
\mu^{0}= \begin{cases}M^{p} \exp a p\left(k^{+}+k^{-}\right), & |x| \leqq L, \\ M^{p} \exp p\left(b^{2} k^{+}+k^{-}\right), & x<-L, \\ M^{p} \exp p\left(k^{+}+b^{2} k^{-}\right) & x>L .\end{cases}
$$

Here $a$ and $b$ are constants to be chosen. Since $D_{t} k^{+}=-v, D_{t} k^{-}=-w$, we have

$$
D_{t} \mu^{0}=-a p \mu^{0}(v+w), \quad|x| \leqq L .
$$

Inserting this in (3.3) we find for $|x| \leqq L$,

$$
D_{t}\left(4 \mu^{0} z^{p}\right)=-4 a p \mu^{0}(v+w) z^{p}+2 p \mu^{0} z^{p-1} v w-2 p \mu^{0} z^{p+1} .
$$

Since $\mu^{ \pm} \leqq M^{p} \leqq \mu^{0}$, the last term will dominate the positive terms in (3.7), (3.8) in the sum (3.4). To handle the growth term in the $z$-equation, let $r^{2}=v w$. We could use Young's inequality as above to write

$$
z^{p-1} v w=\left(z^{p-1} r\right) \cdot r \leqq \frac{1}{p} r^{p+1}+\frac{p-1}{p} z^{p} r,
$$

but it will be better to avoid a factor of $\mu^{0}$ on the $r^{p+1}$ term so that it can be controlled by the last terms in (3.7), (3.8). Thus

$$
\mu^{0} z^{p-1} r \leqq \frac{1}{p} r^{p}+\frac{p-1}{p}\left(\mu^{0}\right)^{p^{\prime}(p-1)} z^{p}
$$

and

$$
2 p \mu^{0} z^{p-1} v w=2 p r \cdot \mu^{0} z^{p-1} r \leqq 2 r^{p+1}+(p-1)(2 r)\left(\mu^{0}\right)^{p /(p-1)} z^{p} .
$$

For the first term we have

$$
2 r^{p+1}=2 r^{p-1} r^{2}=2\left(v^{p-1} w^{p-1}\right)^{1 / 2}(v w) \leqq\left(v^{p-1}+w^{p-1}\right) v w=v^{p} w+v w^{p} .
$$

Since $\mu^{ \pm} \geqq 1$, this term is bounded by the corresponding terms in (3.7), (3.8).

We now will have shown that $R \leqq 0$ in (3.4) for $|x| \leqq L$ provided the second term in (3.13) is controlled by the $\left(D_{t} \mu^{0}\right)$-term, i.e., if

$$
(p-1)(2 r)\left(\mu^{0}\right)^{p /(p-1)} z^{p} \leqq 4 a p \mu^{0}(v+w) z^{p} .
$$

Since $2 r \leqq v+w$, this holds if

or

$$
\left(\mu^{0}\right)^{1 /(p-1)} \leqq 4 a
$$

$$
M^{p /(p-1)} \exp \left\{\frac{p}{p-1} a\left(k^{+}+k^{-}\right)\right\} \leqq a .
$$

According to Lemma 2.2, for given $\varepsilon_{1}$, there is a time $t_{0}$ so that

$$
k^{+}+k^{-} \leqq \varepsilon_{1} \quad \text { for }|x| \leqq L, \quad t \geqq t_{0} .
$$


We choose $\varepsilon_{1}$ below in terms of the mass $m$. satisfy

Assume that $p \geqq 2$, so that $p /(p-1) \leqq 2$. To verify (3.14), it will be sufficient to

$$
M^{2} e^{2 a \varepsilon_{1}} \leqq 4 a .
$$

Take $a=e M^{2} / 4$, so that the inequality becomes $\exp \left(2 a \varepsilon_{1}\right) \leqq e$. We are thus led to choose $\varepsilon_{1}$ so that $2 a \varepsilon_{1}=1$, or

$$
\varepsilon_{1}=1 / 2 a=2 e^{-1} M^{-2} .
$$

With this choice of $\varepsilon_{1},(3.16)$, and therefore (3.14), is satisfied. We have therefore established that

$$
R(x, t) \leqq 0 \quad \text { for }|x| \leqq L, \quad t \geqq t_{0} .
$$

Here $t_{0}$ depends on $L$ and our choice of $\varepsilon_{1}$.

We now show that we can also ensure $R \leqq 0$ for $x<-L$ if $\varepsilon_{0}$ and $b$ are chosen properly. Recalling (3.10), we have in place of (3.11), for $x<-L, D_{t} \mu^{0}=$ $-p \mu^{0}\left(b^{2} v+w\right)$. We estimate as before, except that (3.14) is replaced by

$$
(p-1)(2 r)\left(\mu^{0}\right)^{p /(p-1)} z^{p} \leqq 4 p \mu^{0}\left(b^{2} v+w\right) z^{p} .
$$

To establish this inequality, we first write $2 r \leqq b v+w / b=\left(b^{2} v+w\right) / b$. Thus (3.19) will hold provided $\left(\mu^{0}\right)^{1 /(p-1)} \leqq 4 b$. Now (3.9) and Lemma $2.3($ a) imply that

$$
k^{+} \leqq \varepsilon_{0} \quad \text { for } x<-L, \quad k^{-} \leqq \varepsilon_{0} \quad \text { for } x>L,
$$

and $k^{-} \leqq m$ always. Therefore

$$
\mu^{0} \leqq M^{p} \cdot \exp \left(p b^{2} \varepsilon_{0}\right) \cdot M^{p}
$$

thus (3.19) will be implied by

$$
M^{2 p /(p-1)} \exp \left(\frac{p}{p-1} b^{2} \varepsilon_{0}\right) \leqq 4 b,
$$

and for $p \geqq 2$ by $M^{4} \exp \left(2 b^{2} \varepsilon_{0}\right) \leqq 4 b$. Thus if we choose $b=e M^{4} / 4$ and

$$
\varepsilon_{0}=1 / 2 b^{2}=8 e^{-2} M^{-8},
$$

then (3.19) is satisfied. We have shown that $R \leqq 0$ for $x<-L$ and $t \geqq 0$. In just the same way, $R \leqq 0$ for $x>L, t \geqq 0$.

We have now established that $R \leqq 0$ for $t \geqq t_{0},-\infty<x<\infty$. In fact, with $p \geqq 2$ our estimates show that $R \leqq-2 \mu^{0} z^{p+1}$, and integrating (3.4) in $x$ gives

$$
\frac{d}{d t} \int_{-\infty}^{\infty}\left(\mu^{+} v^{p}+\mu^{-} w^{p}+4 \mu^{0} z^{p}\right) d x+2 \int_{-\infty}^{\infty} \mu^{0} z^{p+1} d x \leqq 0 .
$$

Define $Y(t), N(t)$ by

$$
\begin{aligned}
& Y(t)^{p}=\int_{-\infty}^{\infty}\left(\mu^{+} v^{p}+\mu^{-} w^{p}+4 \mu^{0} z^{p}\right) d x, \\
& N(t)^{p}=\int_{-\infty}^{\infty}\left(v^{p}+w^{p}+4 z^{p}\right) d z .
\end{aligned}
$$


Our differential inequality yields

$$
Y(t)^{p}+2 \int_{t_{0}}^{t} \int_{-\infty}^{\infty} \mu^{0} z^{p+1} d x d t \leqq Y\left(t_{0}\right)^{p} .
$$

Now $\mu^{0}, \mu^{ \pm} \geqq 1$, so that $N(t) \leqq Y(t)$ for each $t$. Also $\mu^{ \pm} \leqq M^{p}$. We choose $a, \varepsilon_{1}$ so that $a\left(k^{+}+k^{-}\right) \leqq a \varepsilon_{1} \leqq 1 / 2$ and thus $\mu_{0} \leqq M^{p} e^{p / 2}$ for $|x| \leqq L, t \geqq t_{0}$. Similarly for $|x| \geqq L$, $b^{2} \varepsilon_{0} \leqq 1 / 2$ and $\mu^{0} \leqq M^{p} \exp p(m+1 / 2)=M^{2 p} e^{p / 2}$. In any case we have

$$
1 \leqq \mu^{ \pm}, \mu^{0} \leqq M_{1}^{p}, \quad M_{1} \equiv M^{2} e^{1 / 2}=\exp (2 m+1 / 2),
$$

and therefore $Y(t) \leqq M_{1} N(t)$. Let

$$
K_{1}=\sup \left\{v, w, z:-\infty<x<\infty, t=t_{0}\right\} .
$$

Then

$$
Y\left(t_{0}\right)^{p} \leqq M_{i}^{p} K_{1}^{p-1} \int(v+w+4 z) d x=m M_{1}^{p} K_{1}^{p-1} .
$$

One consequence of (3.22), which we note for later use, is

$$
\int_{0}^{\infty} \int_{-\infty}^{\infty} z^{p+1} d x d t<\infty, \quad p \geqq 2 .
$$

Also

$$
N(t) \leqq Y(t) \leqq Y\left(t_{0}\right) \leqq M_{1} K_{1}\left(m / K_{1}\right)^{1 / p} .
$$

Finally we let $p \rightarrow \infty . N(t)$ converges to the supremum of $v, w, z$ at time $t$, and we obtain

$$
v(x, t), w(x, t), z(x, t) \leqq M_{1} K_{1}, \quad t \geqq t_{0} .
$$

According to the Existence Theorem, $v, w, z$ are bounded for $0 \leqq t \leqq t_{0}$, and it follows that they are bounded uniformly for $0 \leqq t<\infty, x \in \mathbb{R}$.

\section{Asymptotics and Stability in $L^{p}$}

In this section we show that the interaction terms are integrable in space-time and prove Theorem 2 as a consequence. We then prove Theorems 3 and 4 . We first show that the $v$-mass decays on the left, and $w$-mass on the right, and the $z$-mass everywhere.

Lemma 4.1. For any $x \in \mathbb{R}$,

$$
\begin{aligned}
& \int_{-\infty}^{x} v\left(x^{\prime}, t\right) d x^{\prime} \rightarrow 0 \quad \text { as } \quad t \rightarrow \infty, \\
& \int_{x}^{\infty} w\left(x^{\prime}, t\right) d x^{\prime} \rightarrow 0 \quad \text { as } \quad t \rightarrow \infty .
\end{aligned}
$$

Also

$$
\int_{-\infty}^{\infty} z\left(x^{\prime}, t\right) d x^{\prime} \rightarrow 0 \quad \text { as } \quad t \rightarrow \infty
$$


Proof. As remarked in Lemma 2.3(b),

$$
h^{-}(x, t)=\int_{-\infty}^{x}(v+2 z)\left(x^{\prime}, t\right) d x^{\prime}
$$

is nonincreasing in $t$. Therefore it has a limit as $t \rightarrow \infty$. We show that the limit must be zero. Given $\varepsilon$, we choose $a \leqq x$ so that

$$
\int_{-\infty}^{a}\left(v_{0}+2 z_{0}\right) d x^{\prime}<\varepsilon / 2
$$

Then

$$
\int_{-\infty}^{a}(v+2 z)\left(x^{\prime}, t\right) d x^{\prime}<\varepsilon / 2
$$

for all $t \geqq 0$. By Lemma 2.1 ,

$$
\int_{0}^{\infty} \int_{a}^{x} v\left(x^{\prime}, t\right) d x^{\prime} d t<\infty
$$

Also from (3.23),

$$
\int_{0}^{\infty} \int_{-\infty}^{\infty} z^{3}\left(x^{\prime}, t\right) d x^{\prime} d t<\infty
$$

so that by Hölder's inequality

$$
\int_{0}^{\infty}\left[\int_{a}^{x} z\left(x^{\prime}, t\right) d x^{\prime}\right]^{3} d t<\infty .
$$

Consequently there is a time $t$ so that

$$
\int_{a}^{x}(v+2 z)\left(x^{\prime}, t\right) d x^{\prime}<\varepsilon / 2
$$

and $h^{-}(t)<\varepsilon$. Therefore $\lim h^{-}(t)=0$. A similar argument shows that

$$
h^{+}(x, t)=\int_{x}^{\infty}(w+2 z)\left(x^{\prime}, t\right) d x^{\prime}
$$

goes to zero. The conclusions follow from these two statements.

We note one consequence of Lemma 4.1. If we apply the divergence theorem once again to (2.4), we have

$$
h^{-}(x, t)=\int_{t}^{T} v\left(x, t^{\prime}\right) d t^{\prime}+h^{-}(x, T) .
$$

Letting $T \rightarrow \infty$, we find

Similarly

$$
h^{-}(x, t)=\int_{t}^{\infty} v\left(x, t^{\prime}\right) d t^{\prime} .
$$

$$
h^{+}(x, t)=\int_{t}^{\infty} w\left(x, t^{\prime}\right) d t^{\prime} .
$$




\section{Lemma 4.2.}

$$
\int_{0}^{\infty} \int_{-\infty}^{\infty}\left(z^{2}+v w\right) d x d t<\infty
$$

Proof. We estimate with multipliers as in the previous section, this time separately for $x \leqq 0$ and $x \geqq 0$. In the first case we multiply the $w$-equation by $\mu^{-}=\exp 2 h^{-}$and the $z$-equation by 8 , with $h^{-}$as in (4.1). Using (4.3), we have $D_{x} h^{-}=v+2 z, D_{t} h^{-}=$ $-v, D^{-} h^{-}=-2(v+z)$, and $D^{-} \mu^{-}=-4(v+z) \mu^{-}$. At large time, say $t \geqq t_{0}$, we have $1 \leqq \mu^{-} \leqq 2$ for $x \leqq 0$ by Lemma 4.1 . The equations are

$$
\begin{aligned}
D_{t} v+D_{x} v & =z^{2}-v w \\
D_{t}\left(\mu^{-} w\right)-D_{x}\left(\mu^{-} w\right) & =\mu^{-} z^{2}-5 \mu^{-} v w-4 \mu^{-} z w \\
D_{t}(8 z) & =4 v w-4 z^{2} .
\end{aligned}
$$

Integrating over $-\infty<x \leqq 0$, we have

$$
\begin{aligned}
D_{t} & \int_{-\infty}^{0}\left(v+\mu^{-} w+8 z\right) d x+v(0, t)-\left(\mu^{-} w\right)(0, t) \\
& =\int_{-\infty}^{0}\left[\left(-3+\mu^{-}\right) z^{2}+\left(3-5 \mu^{-}\right) v w-4 \mu^{-} z w\right] d x \\
& \leqq-\int_{-\infty}^{0}\left(z^{2}+v w\right) d x
\end{aligned}
$$

for $t \geqq t_{0}$. We now integrate in $t$ to obtain for $t_{1} \geqq t_{0}$,

$$
\begin{aligned}
& \int_{-\infty}^{0}\left(v+\mu^{-} w+8 z\right)\left(x, t_{1}\right) d x+\int_{t_{0}}^{t_{1}} \int_{-\infty}^{0}\left(z^{2}+v w\right) d x d t \\
& \leqq \int_{-\infty}^{0}\left(v+\mu^{-} w+8 z\right)\left(x, t_{0}\right) d x+\int_{t_{0}}^{t_{1}}\left(\mu^{-} w\right)(0, t) d t \leqq 4 m
\end{aligned}
$$

using Lemma 2.1. This shows $z^{2}+v w$ is integrable on $-\infty<x \leqq 0,0 \leqq t<\infty$. For, $x \geqq 0$ the argument is similar; we multiply the $v$-equation by $\mu^{+}=\exp 2 h^{+}, h^{+}$as in (4.2), and the $z$-equation by 8 , and then use the fact that $D^{+} \mu^{+}=-4(w+z) \mu^{+}$.

Proof of Theorem 2. (Limiting Behavior in $L^{p}$ ). The asymptotic description of the solution in $L^{1}(\mathbb{R})$ is an easy consequence of the last two lemmas. We have already seen in Lemma 4.1 that $z(\cdot, t) \rightarrow 0$ in $L^{1}(\mathbb{R})$ as $t \rightarrow \infty$. As observed in [15], the limiting behavior of the other components follows directly from the integrability of the interaction terms: Let $F(x, t)=z^{2}-v w$, and define $v^{*}(x, t)=v(x+t, t)$. Then $D_{t} v^{*}(x, t)=D^{+} v(x+t, t)=F(x+t, t) \equiv F^{*}(x, t)$. Regarding $F^{*}$ as an element of $L^{1}\left(0, \infty ; L^{1}(\mathbb{R})\right)$, we can write

$$
v^{*}(\cdot, t)=v_{0}+\int_{0}^{t} F^{*}(\cdot, s) d s
$$

and it is clear that $v^{*}(\cdot, t)$ converges in $L^{1}(\mathbb{R})$ to a limit $v^{\infty}$. Since

$$
m^{+}=\int_{-\infty}^{\infty}(v+2 z)(x, t) d x
$$


is conserved and $z(\cdot, t) \rightarrow 0$ in $L^{1}$,

$$
\int_{-\infty}^{\infty} v^{\infty}(x) d x=m^{+}
$$

Similar remarks apply to $w$. Convergence in $L^{p}, p<\infty$, follows from convergence in $L^{1}$ and uniform boundedness. Finally,

$$
\left|v^{*}(\cdot, t)\right|_{L^{p}} \leqq K^{(p-1) / p} m^{1 / p},
$$

for each $t$, and therefore the same holds for $v^{\infty}$. Letting $p \rightarrow \infty$, we have $\left|v^{\infty}\right|_{L^{\infty}} \leqq K$.

We now consider solutions $v, w, z$ near a fixed solution $\bar{v}, \bar{w}, \bar{z}$. We first show that solutions which are initially close in $L^{1} \cap L^{\infty}(\mathbb{R})$ remain close for finite time.

Lemma 4.3. Given $T, \varepsilon$, and a solution $\bar{v}, \bar{w}, \bar{z}$, there exists $\delta$ so that if

$$
\left|v_{0}-\bar{v}_{0}\right|_{L^{\infty}}+\left|w_{0}-\bar{w}_{0}\right|_{L^{\infty}}+4\left|z_{0}-\bar{z}_{0}\right|_{L^{\infty}}<\delta,
$$

and the same for $L^{1}$ norms, then for $0 \leqq t \leqq T$,

$$
|v(\cdot, t)-\bar{v}(\cdot, t)|_{L^{\infty}}<\varepsilon, \quad|v(\cdot, t)-\bar{v}(\cdot, t)|_{L^{1}}<\varepsilon,
$$

and similarly for $w, z$.

Proof. We first bound the difference in $L^{\infty}$. Let $y(t)$ be the maximum of $|v-\bar{v}|$, $|w-\bar{w}|,|z-\bar{z}|$ up to time $t$. Also let $K$ be the maximum of $\bar{v}, \bar{w}, \bar{z}$ for $0 \leqq t \leqq T$. Integrating the equations for the differences along characteristics we find easily that

$$
y(t) \leqq y(0)+\int_{0}^{t}\left[4 K y(s)+2 y(s)^{2}\right] d s .
$$

As long as $2 y(s) \leqq K$, we have $y(t) \leqq y(0) e^{5 K T}$ by Gronwall's inequality. If $y(0) \leqq$ $K e^{-5 K T} / 4 \leqq \delta_{0}$, this argument holds for $0 \leqq t \leqq T$. We obtain the conclusion by taking $\delta \leqq \varepsilon e^{-5 K T}$ as well as $\delta \leqq \delta_{0}$.

Next we estimate the difference in $L^{1}$. By the above, the maximum of $v, w, z$ for $t \leqq T$ is $\leqq 2 K$ for $v_{0}, w_{0}, z_{0}$ in some $L^{\infty}$-neighborhood of $\bar{v}_{0}, \bar{w}_{0}, \bar{z}_{0}$. Let

$$
r(t)=|v(t)-\bar{v}(t)|_{L^{1}}+|w(t)-\bar{w}(t)|_{L^{1}}+|z(t)-\bar{z}(t)|_{L^{1}}
$$

A straightforward estimate gives

$$
\left|D_{t}(v-\bar{v})\right|_{L^{1}}+\left|D_{t}(w-\bar{w})\right|_{L^{1}}+\left|D_{t}(z-\bar{z})\right|_{L^{1}} \leqq 15 \operatorname{Kr}(t),
$$

and this differential inequality implies that $r(t) \leqq r(0) \exp (15 K T)$. (E.g., see [16], Sect. I.6; the arguments given there apply to Banach-space-valued functions.)

Proof of Theorem 3. ( $L^{p}$ Stability). Given $\theta$, to be determined later in terms of $\varepsilon$, there exists a time $t_{0}$, according to Lemma 4.1, so that

$$
\int_{-\infty}^{0}(\bar{v}+2 \bar{z}) d x<\theta, \quad \int_{0}^{\infty}(\bar{w}+2 \bar{z}) d x<\theta
$$

For initial data sufficiently close to $\bar{v}_{0}, \bar{w}_{0}, \bar{z}_{0}$ in $L^{1} \cap L^{\infty}$ the solutions are within $\theta$ of 
$v, w, \bar{z}$ in $L^{1}(\mathbb{R})$ for $0 \leqq t \leqq t_{0}$. Then

$$
\int_{-\infty}^{0}(v+2 z) d x<2 \theta, \quad \int_{0}^{\infty}(w+2 z) d x<2 \theta,
$$

at $t=t_{0}$, and therefore for all later time, since the integrals are nonincreasing. In particular

$$
\int_{-\infty}^{\infty} z d x<2 \theta, \quad t \geqq t_{0}
$$

If $\mu^{ \pm}$are defined as in Lemma 4.2 and $\theta \leqq(\log 2) / 4$, then $\mu^{-} \leqq 2$ for $x \leqq 0, \mu^{+} \leqq 2$ for $x \geqq 0$, and the argument of the lemma applies. The time $t_{0}$ depends on $\theta$ but is the same for all solutions under consideration. Rearranging (4.5), we have for $t_{1} \geqq t_{0}$,

$$
\left.\int_{t_{0}}^{t_{1}} \int_{-\infty}^{0}\left(z^{2}+v w\right) d x d t \leqq-\int_{-\infty}^{0}\left(v+\mu^{-} w+8 z\right)(x, t) d x\right]_{t_{0}}^{t_{1}}+\int_{t_{0}}^{t_{1}}\left(\mu^{-} w\right)(0, t) d t
$$

The analogous estimate for $x \geqq 0$ is

$$
\left.\int_{t_{0}}^{t_{1}} \int_{0}^{\infty}\left(z^{2}+v w\right) d x d t \leqq-\int_{0}^{\infty}\left(\mu^{+} v+w+8 z\right)(x, t) d x\right]_{t_{0}}^{t_{1}}+\int_{t_{0}}^{t_{1}}\left(\mu^{+} v\right)(0, t) d t .
$$

We now add these two expressions and use the conservation of mass to obtain

$$
\begin{aligned}
\int_{t_{0}}^{t_{1}} \int_{-\infty}^{\infty}\left(z^{2}+v w\right) d x d t \leqq & \int_{-\infty}^{0}\left(\rho^{-} w\right)\left(x, t_{0}\right) d x+\int_{0}^{\infty}\left(\rho^{+} v\right)\left(x, t_{0}\right) d x \\
& +4 \int_{-\infty}^{\infty} z\left(x, t_{0}\right) d x+\int_{t_{0}}^{t_{1}}\left(\mu^{-} w+\mu^{+} v\right)(0, t) d t
\end{aligned}
$$

with $\rho^{ \pm}=\mu^{ \pm}-1 \geqq 0$. We check that each term on the right is of order $\theta$ : First, for $\theta \leqq(\log 2) / 4$ as above, $\rho^{ \pm} \leqq 8 \theta$, so that each of the first two terms is bounded by $8 \theta \mathrm{m}$. Here $m$ is an upper bound for the mass in the set of solutions considered. According to (4.7), the $z$-term is bounded by $8 \theta$. Finally,

$$
\int_{t_{0}}^{t_{1}} \mu^{-} w(0, t) d t \leqq 2 \int_{t_{0}}^{\infty} w(0, t) d t=2 h^{+}\left(0, t_{0}\right) \leqq 4 \theta,
$$

recalling (4.4), and similarly for $\mu^{+} v$. In summary,

$$
\int_{t_{0}}^{t_{1}} \int_{-\infty}^{\infty}\left(z^{2}+v w\right) d x d t \leqq(16 m+16) \theta \text { for } t_{1} \geqq t_{0} .
$$

As a consequence, with $F=z^{2}-v w$ again,

$$
\int_{t_{0}}^{\infty} \int_{-\infty}^{\infty}|F| d x d t \leqq \delta \equiv(16 m+16) \theta
$$

Recalling from the proof of Theorem 2 that

$$
v^{*}(\cdot, t)-v^{*}\left(\cdot, t_{0}\right)=\int_{t_{0}}^{t} F^{*}(\cdot, t) d t
$$

we see that $\left|v^{*}(\cdot, t)-v^{*}\left(\cdot, t_{0}\right)\right|_{L^{1}} \leqq \delta$ for $t \geqq t_{0}$. Then for $t \geqq t_{0}$ we have, with all norms 
in $L^{1}$,

$$
\begin{aligned}
|v(t)-\bar{v}(t)| & =\left|v^{*}(t)-\bar{v}^{*}(t)\right| \\
& \leqq\left|v^{*}\left(t_{0}\right)-\bar{v}^{*}\left(t_{0}\right)\right|+\left|v^{*}(t)-v^{*}\left(t_{0}\right)\right|+\left|\bar{v}^{*}(t)-\bar{v}^{*}\left(t_{0}\right)\right| \\
& \leqq \theta+2 \delta .
\end{aligned}
$$

This is $<\varepsilon$ if we take $\theta=\varepsilon /(33(m+1))$. The same works for $w$, and the result is verified for $p=1$. For $p>1$, it will follow from the $L^{1}$ case and Theorem 4 .

Proof of Theorem 4. (Boundedness in a Neighborhood). We check that the argument of Sect. 3 can be applied to solutions with initial data close enough to $\bar{v}_{0}, \bar{w}_{0}, \bar{z}_{0}$. Let $\bar{m}$ be the mass of the fixed solution $\bar{v}, \bar{w}, \bar{z}$. In the earlier argument $m$ will now denote an upper bound for the mass in a neighborhood of $\bar{v}, \bar{w}, \bar{z}$, e.g., $\bar{m}+1$. Choose $\varepsilon_{0}, \varepsilon_{1}$ as in (3.21), (3.17); then choose $L$ so that

$$
\int_{|x|>L}\left(\bar{v}_{0}+\bar{w}_{0}+4 \bar{z}_{0}\right) d x<\varepsilon_{0} / 2
$$

and $t_{0}$ so that

$$
\int_{-\infty}^{L}(\bar{v}+2 \bar{z}) d x, \int_{-L}^{\infty}(\bar{w}+2 \bar{z}) d x<\varepsilon_{1} / 2 \text { for } t=t_{0},
$$

using Lemma 4.1. Assume $v_{0}, w_{0}, z_{0}$ close enough to $\bar{v}_{0}, \bar{w}_{0}, \bar{z}_{0}$, so that

$$
\int_{-\infty}^{\infty}\left(\left|v_{0}-\bar{v}_{0}\right|+\left|w_{0}-\bar{w}_{0}\right|+4\left|z_{0}-\bar{z}_{0}\right|\right) d x<\varepsilon_{0} / 2
$$

and

$$
\int_{-\infty}^{\infty}(|v-\bar{v}|+|w-\bar{w}|+4|z-\bar{z}|) d x<\varepsilon_{1} / 2 \quad \text { for } t \leqq t_{0},
$$

using Lemma 4.3. By (4.8), (4.10)

$$
\int_{-\infty}^{-L}\left(v_{0}+2 z_{0}\right)<\varepsilon_{0}, \quad \int_{L}^{\infty}\left(w_{0}+2 z_{0}\right)<\varepsilon_{0},
$$

and since the integrals are nonincreasing,

$$
\int_{-\infty}^{-L}(v+2 z) d x<\varepsilon_{0}, \quad \int_{L}^{\infty}(w+2 z)<\varepsilon_{0}, \quad t \geqq 0 .
$$

By (4.9), (4.11), we have for $|x| \leqq L, t=t_{0}$,

$$
\int_{-\infty}^{x}(v+2 z) d x<\varepsilon_{1}, \quad \int_{x}^{\infty}(w+2 z) d x<\varepsilon_{1}
$$

for $|x| \leqq L, t=t_{0}$. In view of (4.3), (4.4), conditions (4.12) and (4.13) imply (3.20), (3.15), and the argument proceeds without change.

\section{Uniform Asymptotics}

Throughout this section we assume that $v_{0}, w_{0}, z_{0} \rightarrow 0$ as $x \rightarrow \pm \infty$ in addition to the earlier admissibility conditions. $K$ will denote an upper bound for the solution, and 
$F(x, t)=z^{2}-v w$.

Lemma 5.1. Given $\varepsilon$ there exists $A$ so that

$$
\begin{array}{llrl}
v(x, t) \leqq \varepsilon & \text { for } & |x-t| \geqq A, \\
w(x, t) \leqq \varepsilon & \text { for } & |x+t| \geqq A, \\
z(x, t) \leqq \varepsilon & \text { for } & |x| \geqq A .
\end{array}
$$

Proof. Integrating $D^{+} v=z^{2}-v w$ along a characteristic, we have

$$
v(x, t) \leqq v_{0}(x-t)+K \int_{0}^{t} z(x-t+s, s) d s
$$

and to derive the statement for $v$ we will check that

$$
J(y)=\int_{0}^{\infty} z(y+s, s) d s<\delta
$$

when $|y|$ is large. Since $F \in L^{1}(\mathbb{R} \times(0, \infty))$, for $A$ large enough,

$$
\int_{0}^{\infty} \int_{|y|>A}|F(y+t, t)| d y d t<\delta .
$$

If $y \leqq-A$, we integrate $z_{t}=-F / 2$ over $S^{-}=\{(x, t):-\infty<x<y+t, 0<t<T\}$ to obtain

$$
\int_{0}^{T} z(y+t, t) d t+\int_{-\infty}^{Y} z_{0}(x) d x=\int_{-\infty}^{y+T} z(x, T) d x+\frac{1}{2} \iint_{S^{-}} F .
$$

Since the $z$-mass decays, the first integral on the right tends to zero as $T \rightarrow \infty$. Thus $J(y) \leqq \delta / 2$. If $y \geqq A$, we integrate over $S^{+}=\{(x, t): y+t<x<\infty, 0<t<T\}$, obtaining

$$
\int_{0}^{T} z(y+t, t) d t+\int_{y+T}^{\infty} z(x, T) d x=\int_{y}^{\infty} z_{0}(x) d x-\frac{1}{2} \iint_{S^{+}} F .
$$

If $A$ is large enough so that the $z_{0}$-integral is $\leqq \delta / 2$, we have $J(y) \leqq \delta$. The treatment of $w$ is of course similar.

To estimate $z(x, t)$ for $x$ large and positive we write

$$
z(x, t) \leqq z_{0}(x)+K \int_{0}^{t} w(x, s) d s
$$

bounding $v$ by $K$. By Lemma 2.3(a) the integral is small for large enough $x$. For $x$ negative we reverse the roles of $v$ and $w$.

Lemma 5.2. $(v w)(x, t) \rightarrow 0$ as $t \rightarrow \infty$, uniformly in $x \in \mathbb{R}$.

Proof. Given $\varepsilon, v(x, t) \leqq \varepsilon$ for $x-t \leqq-A$ and thus for $x \leqq 0$ when $t \geqq A$. Therefore $v w \leqq K \varepsilon$ for $x \leqq 0, t \geqq A$. Similarly for $x \geqq 0, t \geqq A, w \leqq \varepsilon$ and $v w \leqq K \varepsilon$.

We are now ready to prove Theorem 5 . We first show that $z(x, t) \rightarrow 0$ as $t \rightarrow \infty$, uniformly in $x \in \mathbb{R}$, using the last lemma. Given $\varepsilon$, there is a time $t_{0}$ so that $(v w)(x, t) \leqq \varepsilon^{2}$ for all $t \geqq t_{0}$ and all $x$. By the comparison theorem for ordinary 
differential equations, for each $x, z(x, t) \leqq y(t)$ for $t \geqq t_{0}$, where $y$ is the solution of

$$
y^{\prime}=\varepsilon^{2}-y^{2}, \quad y\left(t_{0}\right)=z\left(x, t_{0}\right) .
$$

If $z\left(t_{0}\right) \leqq \varepsilon$, then $y(t) \leqq \varepsilon$ for all $t \geqq t_{0}$; otherwise

$$
y(t)=\varepsilon \operatorname{coth}\left\{\varepsilon\left(t-t_{0}\right)+\theta\right\}, \quad \theta=\operatorname{coth}^{-1}\left(y\left(t_{0}\right) / \varepsilon\right) .
$$

If $t \geqq t_{0}+\varepsilon^{-1}$, then $y(t) \leqq \varepsilon$ coth 1 . Since $t_{0}$ was independent of $x$, it follows that $z \rightarrow 0$ uniformly.

We now show that $v, w$ converge uniformly in the characteristic directions, following an argument of Tartar [15, Theorem 5]. Integrating the equation $D^{+} v=F$ along characteristics, we have

$$
v(x, t)=v_{0}(x-t)+\int_{0}^{t} F(x-t+s, s)
$$

so that $v(x, t) \leqq g^{+}(x-t)$, where

$$
g^{+}(y)=v_{0}(y)+\int_{0}^{\infty}|F(y+s, s)| d s .
$$

Since $F \in L^{1}, g^{+}(y)$ is finite a.e. and $g^{+} \in L^{1}(\mathbb{R})$. Similarly $w(x, t) \leqq g^{-}(x+t)$ with

$$
g^{-}(y)=w_{0}(y)+\int_{0}^{\infty}|F(y-s, s)| d s,
$$

and $z(x, t) \leqq g^{0}(x)$

$$
g^{0}(x)=z_{0}(x)+\int_{0}^{\infty}|F(x, s)| d s
$$

both $g^{-}$and $g^{0}$ are also $L^{1}$.

These upper bounds will allow us to show that $\{v(\cdot, t)\}$ is uniformly Cauchy. Let $v^{*}(x, t)=v(x+t, t)$ as before; let $T$ be a large time to be chosen. Since $D_{t} v^{*}=$ $F(x+t, t)$, we have for $t \geqq T$.

and

$$
v^{*}(x, t)-v^{*}(x, T)=\int_{T}^{t} F(x+s, s) d s,
$$

$$
\begin{gathered}
\left|v^{*}(x, t)-v^{*}(x, T)\right| \leqq K I_{1}+K I_{2}, \\
I_{1}=\int_{T}^{t} z(x+s, s) d s, \quad I_{2}=\int_{T}^{t} w(x+s, s) d s .
\end{gathered}
$$

By the above,

$$
I_{1} \leqq \int_{T}^{\infty} g^{0}(x+s) d s=\int_{T+x}^{\infty} g^{0}(s) d s \leqq \int_{T}^{\infty} g^{0}(s) d s,
$$

provided $x \geqq-A$. In the same way, also for $x \geqq-A$,

$$
I_{2} \leqq \int_{T}^{\infty} g^{-}(x+2 s) d s \leqq \frac{1}{2} \int_{2 T-A}^{\infty} g^{-}(s) d s
$$

Now given $\varepsilon$, suppose we choose $A$ as in Lemma 5.1. Then $v^{*}(x, t) \leqq \varepsilon$ for $x \leqq-A$, 
$t \geqq 0 ;$ thus

$$
\left|v^{*}(x, t)-v^{*}(x, T)\right| \leqq \varepsilon, \quad x \leqq-A, \quad t \geqq 0 .
$$

On the other hand, for $x \geqq-A,(5.1)$ and (5.2) apply. Since $g^{0}, g^{-} \in L^{1}(\mathbb{R})$, we can choose $T$ large enough so that $I_{1}, I_{2} \leqq \varepsilon / 2 K$, and therefore

$$
\left|v^{*}(x, t)-v^{*}(x, T)\right| \leqq \varepsilon, \quad x \geqq-A, \quad t \geqq T .
$$

This shows that $\left\{v^{*}(\cdot, t)\right\}$ is Cauchy in the $L^{\infty}$ norm and converges, necessarily to the same limit $v^{\infty}$ as before. An analogous argument shows that $w(\cdot, t)$ converges uniformly to $w^{\infty}$.

We have seen in Lemma 5.1 that $v(\cdot, t), w(\cdot, t) \in C_{0}(\mathbb{R})$, the subspace of $L^{\infty}(\mathbb{R})$ consisting of continuous functions which tend to zero as $x \rightarrow \pm \infty$. Since this subspace is closed, $v^{\infty}, w^{\infty} \in C_{0}(\mathbb{R})$ as well. The statement that $v(x, t), w(x, t)$ go to zero uniformly for $x$ in a finite interval follows from this fact and the uniform convergence.

Acknowledgement. It is a pleasure to thank M. Reed for a number of stimulating discussions about these questions.

\section{References}

1. Broadwell, J. E.: Shock structure in a simple discrete velocity gas. Phys. Fluids 7, 1243-47 (1964)

2. Cabannes, H.: Solution globale du problème de Cauchy en théorie cinétique discrète. J. Mec. 17, 1-22

3. Caflisch, R., Papanicolaou, G.: The fluid-dynamical limit of a nonlinear model Boltzmann equation. Commun. Pure Appl. Math. 32, 589-619 (1979)

4. Courant, R., Hilbert, D.: Methods of mathematical physics. Vol. II, New York: Interscience 1962

5. Godunov, S. K., Sultangazin, U. M.: On discrete models of the kinetic Boltzmann equation. Russ. Math. Surv. 26, No. 3, 1-56 (1971)

6. Illner, R., Reed, M. C.: The decay of solutions of the Carleman model. Math. Methods Appl. Sci. 3, $121-127$ (1981)

7. Illner, R.: Global existence results for discrete velocity models of the Boltzmann equation in several dimensions. (Preprint)

8. Illner, R.: The Broadwell model for initial values in $L_{+}^{1}(\mathbb{R})$. Commun. Math. Phys. 93, 341-353 (1984)

9. Inoue, K., Nishida, T.: On the Broadwell model of the Boltzmann equation for a simple discrete velocity gas. Appl. Math. Optim. 3, 27-49 (1976)

10. Kaniel, S., Shinbrot, M.: The Boltzmann equation, II: Some discrete velocity models. (to appear in J. Mec.)

11. Kawashima, S.: Global solution of the initial value problem for a discrete velocity model. Proc. Jpn. Acad. 57, 19-24 (1981)

12. Leguillon: Solution globale d'un problème avec conditions aux limites en theorie cinetique discrète. C.R. Acad. Sci. Paris $A-B$ 285, 1125-28 (1977)

13. Nishida, T., Mimura, M.: On the Broadwell's model for a simple discrete velocity gas. Proc. Jpn. Acad. 50, 812-17 (1974)

14. Tartar, L.: Existence globale pom un systeme hyperbolique semilinéarie de la théorie cinetique des gaz. Sémin. Goulaouic-Schwarz, No. $1(1975 / 76)$

15. Tartar, L.: Some existence theorems for semilinear hyperbolic systems in one space variable, MRC Technical Summary Report, University of Wisconsin (1980)

16. Hale, J. K.: Ordinary differential equations. New York: Wiley-Interscience 1969

Communicated by J. L. Lebowitz

Received February 5, 1985 
